\title{
Kubelka-Munk or Neural Networks for Computer Colorant Formulation?
}

\author{
Stephen Westland ${ }^{* a}$, Laura Iovine ${ }^{\mathrm{a}}$ and John M Bishop ${ }^{\mathrm{b}}$ \\ ${ }^{a}$ Colour \& Imaging Institute, Derby University, Derby, UK; \\ ${ }^{\mathrm{b}}$ Department of Cybernetics, Reading University, UK.
}

\begin{abstract}
Traditionally Computer Colorant Formulation has been implemented using a theory of radiation transfer known as Kubelka-Munk (K-M) theory. Kubelka-Munk theory allows the prediction of spectral reflectance for a mixture of components (colorants) that have been characterised by absorption $K$ and scattering $S$ coefficients. More recently it has been suggested that Artificial Neural Networks (ANNs) may be able to provide alternative mappings between colorant concentrations and spectral reflectances and, more generally, are able to provide transforms between colour spaces. This study investigates the ability of ANNs to predict spectral reflectance from colorant concentrations using a set of data measured from known mixtures of lithographic printing inks. The issue of over-training is addressed and we show that the number of hidden units in the network must be carefully selected. We show that it is difficult to train a conventional neural network to the level that matches the performance that can be achieved using the K-M theory. However, a hybrid model is proposed that may out-perform the K-M model.
\end{abstract}

Keywords: colour, Kubelka-Munk theory, artificial neural networks, colour recipe prediction.

\section{INTRODUCTION}

Traditionally Computer Colorant Formulation has been implemented using a theory of radiation transfer known as Kubelka-Munk (K-M) theory ${ }^{1-4}$. Kubelka-Munk theory allows the prediction of spectral reflectance for a mixture of components (colorants) that have been characterised by absorption $K$ and scattering $S$ coefficients. It has been shown that the Kubelka-Munk coefficients $K$ and $S$ are related to, but not equal to, the fundamental optical coefficients for absorption $\varepsilon$ and scattering $\sigma^{4}$. More recently it has been suggested that Artificial Neural Networks (ANNs) may be able to provide alternative mappings between colorant concentrations and spectral reflectances ${ }^{5-7}$ and, more generally, are able to provide transforms between colour spaces ${ }^{8-9}$. This study addresses two key issues; firstly, it presents a quantitative comparison of K-M theory and ANNs for a given problem domain; secondly, it suggests that significant advances may be made by combining both K-M and ANNs to form a hybrid ANN-KM model. Quantitative data are presented for the prediction of spectral reflectance for a set of known mixtures of printing inks.

\subsection{Kubelka-Munk model}

The Kubelka-Munk theory characterises colorants according to two coefficients, $K$ and $S$, the absorption and scattering coefficients respectively. The K-M theory is a two-flux version of a multi-flux method for solving radiation-transfer problems. Although more exact theories exist ${ }^{10}$ the continued use of the K-M theory is due to its simplicity and the ease by which the coefficients $K$ and $S$ can be estimated. The application of K-M theory varies depending upon whether the application is for the prediction of the colour of textiles, (opaque) paints or (translucent) printing inks. The version of the theory that is considered in this study is that designed for transluscent printing inks where the values of $K$ and $S$ must each be determined absolutely ${ }^{11}$. Using this model, the $K_{i}$ and $S_{i}$ contributions for each colorant $i$ are assumed to be additive for mixture $j$ thus

$K_{j}=\Sigma_{i} c_{i j} K_{i}$ and

$S_{j}=\sum_{i} C_{i j} S_{i}$

\footnotetext{
*For correspondence contact s.westland@derby.ac.uk
} 
where $K_{j}$ and $S_{j}$ are the $K$ and $S$ values for mixture $j, c_{i j}$ is the concentration of colorant $i$ in mixture $j$, and $K_{i}$ and $S_{i}$ are the $K$ and $S$ coefficients for colorant $j$. Of course, equation 1 is repeated for each wavelength interval so that $\mathrm{K}_{j}, \mathrm{~S}_{j}, K_{i}$, and $S_{i}$ are all functions of wavelength. The values of $K_{j}$ and $S_{j}$ need to be computed from measurements of the reflectance of the mixture. Many methods are available to determine the values of $K_{i}$ and $S_{i}$ but the method used in this study involves computing $K_{i}$ and $S_{i}$ from two measurements of each mixture; a measurement of the ink printed over white and a measurement of the ink printed over black. Once the values of $K_{i}$ and $S_{i}$ are known at each wavelength and for each colorant $i$, equation 1 can be used to predict the $K$ and $S$ values of any mixture defined by a concentration vector $\mathbf{c}$ and the reflectance $R_{j}$ of the mixture can then be easily computed.

\subsection{The Neural Network model}

The K-M theory allows a mapping between a colorant vector $\mathbf{c}$ and a reflectance vector $\mathbf{r}$ that defines the colour-prediction problem. A class of ANNs known as multi-layer perceptrons (MLPs) have been shown to be capable of approximating any continuous function to any degree of accuracy ${ }^{13}$. An MLP is a layered structure of simple processing units. The units in the first or input layer take their input from a real-world vector and the output of the units in the last or output layer provide the output of the network. There may be one or more hidden layers of units between the input and output layer. Most MLPs are fully connected; that is, each unit provides a weighted input to each unit in the next layer. Each unit in the network is also associated with a transfer function that maps the input of the unit to its output and many different transfer functions are available. Information is thus processed from the input layer to the output layer in order to perform a mapping from an input vector $\mathbf{i}$ to an output vector $\mathbf{o}$. MLPs can learn to perform an arbitrary mapping if they are presented with sufficient examples of the mapping problem $\mathbf{i} \rightarrow \mathbf{o}$. Learning, in an MLP, is a process of optimisation (during which changes are made to the weights in the network) to minimise the RMS error between the desired output vector $\mathbf{o}_{\mathrm{t}}$ and the actual vector $\mathbf{0}_{\mathbf{a}}$. MLPs thus require a training set of input-output pairs. Once suitably trained, however, the network can perform the mapping $\mathbf{i} \rightarrow \mathbf{o}$ for input vectors $\mathbf{i}$ that were not used during the training of the network - this important property is known as generalisation. The Kolmogorov theorem ${ }^{12}$ states that a single hidden layer of units is sufficient to solve any problem. Thus, a three-layer network can learn the colour-prediction problem $\mathbf{c} \rightarrow \mathbf{r}$. However, although the dimensionality of the input and output layers is determined by the problem (specifically by the length of the vectors $\mathbf{c}$ and $\mathbf{r}$ ) the number of hidden units that are required to solve any given problem can only be determined empirically. Further, the choice of transfer function, learning rule (for changing the weights), and the number of training examples required must all be determined empirically.

\section{METHODOLOGY}

\subsection{Printing ink set}

Experiments were conducted using a set of lithographic printing inks printed onto leneta opacity charts. A set of six components (clear resin, white, black, cyan, magenta and yellow) were used and 108 unique recipes were prepared and printed over white and black portions of the opacity charts. The spectral reflectance values for each sample were measured (over white and over black) using a commercial reflectance spectrophotometer at $10 \mathrm{~nm}$ intervals in the range 400-700 $\mathrm{nm}$. An additional set of reductions with the clear resin were additionally produced to calibrate the K-M model.

\subsection{Characterisation of Kubelka-Munk model}

For each colorant in the set a number of pairs (over white and over black) of reflectance measurements were used to determine $K$ and $S$ at each wavelength for a range of colorant concentrations. Saunderson correction values (internal 0.60, external 0.04) were applied to all measured reflectance values before use in computations. The reflectance (over white substrate only) of each of 33 of the known mixtures was predicted and compared with the known reflectance for that mixture. Prediction performance was quantified using computations of CIELAB $\Delta \mathrm{E}$ between the known and predicted spectra using illuminant D65.

\subsection{Training of Neural-Network model}

The neural networks were used to approximate a function between a colorant vector c describing six components (pigments) and a reflectance vector $\mathbf{r}$ containing 31 reflectance values $(400-700 \mathrm{~nm})$. Therefore the topology of the MLPs chosen for the colour prediction problem require 6 input units, each corresponding to the concentrations of one of the six printing inks used in the mixture. The output layer requires 31 units, each corresponding to reflectance at $400 \mathrm{~nm}, 410 \mathrm{~nm}$, $430 \mathrm{~nm} \ldots . .700 \mathrm{~nm}$. A training set of 75 mixtures was used to train the neural networks and the performance of the networks was tested on a set of 33 test mixtures. Prediction performance was quantified both using standard measures of 
root-mean square RMS error and computations of CIELAB $\Delta \mathrm{E}$ between the known and predicted spectra using illuminant D65.

Networks were trained using the back-propagation algorithm with momentum. The algorithm (implemented by the training function traingdm in Matlab) requires two parameters: the learning rate and momentum term. The momentum term was fixed at 0.9 and the learning rate was fixed at 0.2 . Networks were trained using 5, 10, 15, and 20 units in the hidden layer each for a total of 500,000 epochs. One epoch is a term used to describe the case where the samples in the training set are each presented to the network, the errors at the output layer computed, and small changes made to the weights in the network according to the back-propagation algorithm. Each network was trained six times starting each time with a randomly chosen set of weights. We chose the Tan-Sigmoid transfer function for units in the hidden layer and the LogSigmoid function for units in the output layer.

The network reached the best performance with 10 units in the hidden layer. A modified version of the algorithm was then used whereby the learning rate linearly reduced during training from 0.2 to 0.05 (algorithm implemented by the training function traingdx). This network was trained four times each from different random starting weights.

\section{RESULTS}

The neural network was trained six times for each condition and the average RMS errors are shown in Figure 1 (for the training set) and Figure 2 (for the test set) each for different numbers of units in the hidden layer. As expected the error for the training set decreased with increasing numbers of units in the hidden layer. However, the ability of the network to generalise can only be tested by a separate data set that was not used during training. Thus, Figure 2 shows that when the number of hidden units exceeds 10, the error on the test set begins to increase. This is evidence of over-training and indicates that the networks is starting to learn the noise in the training set rather than the relationship between colorant concentration and reflectance.

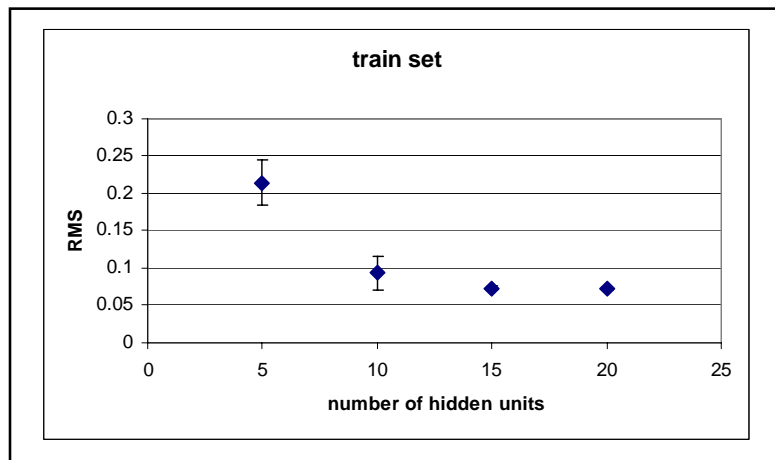

Figure 1: RMS error for training set

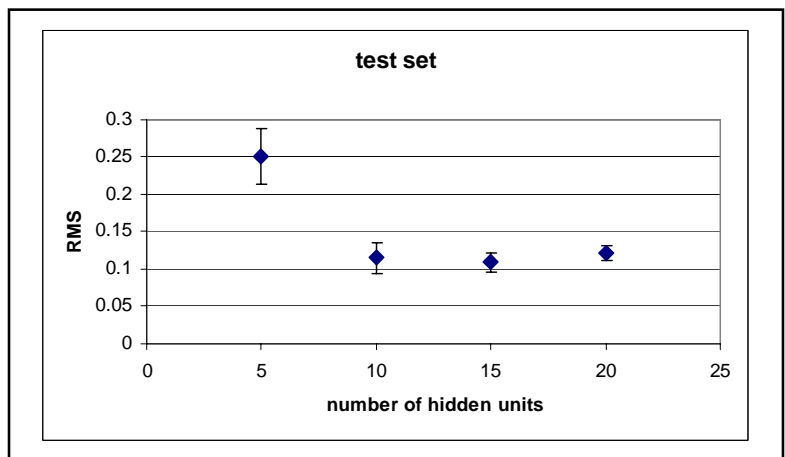

Figure 2: RMS error for test set

The data shown in Figures 1 and 2 suggest that 10 hidden units are appropriate for this particular problem and that RMS errors of about 0.1 can be achieved on the test set. However, there are other parameters such as the choice of activation function, the training algorithm, and the learning rate that will affect performance. Consequently, a network was trained with 10 hidden units using an algorithm that allows the learning rate to be reduced during training. Results are shown for four trials (each starting from different random values for the weights) of this network in Table 1.

Table 1 shows that the use of a learning rate that reduces during training produces an average RMS error of 0.05 . Nevertheless, if the performance of the networks is recast in terms of a CIELAB $\Delta \mathrm{E}$ value then the average $\Delta \mathrm{E}$ between actual and predicted reflectance for the test set is 8.23 units which is much greater than the value of 2.9 that can be obtained using the K-M model. 


\begin{tabular}{|rrr|}
\hline Trial & training set & test error \\
\hline 1 & 0.0373 & 0.0665 \\
2 & 0.0262 & 0.0743 \\
3 & 0.0358 & 0.0455 \\
4 & 0.0281 & 0.0304 \\
\hline mean & $\mathbf{0 . 0 3 1 9}$ & $\mathbf{0 . 0 5 4 2}$ \\
stdev & 0.0048 & 0.0173 \\
\hline
\end{tabular}

Table 1: RMS errors for networks trained using varying learning rates during training.

\section{DISCUSSION}

The results presented show that although in principle ANNs can learn to map between colorant concentrations and spectral reflectance, in practice the optimisation of the various parameters of the network is difficult and consequently it is not easy for the ANN to out-perform the K-M model. We have demonstrated that the proper use of separate training and test sets is essential in order to correctly assess the generalisation performance of trained networks. Experiments are underway that utilise a hybrid model based upon an ANN but maintaining some key features of the K-M model. Initial results suggest that this model can outperform both ANN and K-M approaches. These experiments are still underway and data are not available at this time. Results from these experiments will, however, be included in our presentation at AIC01.

\section{ACKNOWLEDGEMENTS}

This work is supported by Colourware Ltd (www.colourware.co.uk).

\section{REFERENCES}

1. P. Kubelka, "New Contributions to the Optics of Intensely Light-Scattering Materials Part I”, JOSA, 38 (5), pp. 448451, 1948.

2. P. Kubelka, "New Contributions to the Optics of Intensely Light-Scattering Materials Part II: Nonhomogeneous Layers”, JOSA, 44 (4), pp. 330-335, 1954.

3. E. Allen, "Prediction of Optical Properties of Paints from Theory”, Journal of Paint Technology, 45 (584), pp. 65-72, 1973.

4. J.H. Nobbs, "Kubelka-Munk theory and the prediction of reflectance”, Review of Progress in Coloration (SDC), 15, pp. 66-75, 1985.

5. JM Bishop, MJ Bushnell and S. Westland S, “Application of neural networks to computer recipe prediction”, CRA, 16 (1), 3-9, 1991.

6. S. Westland, J.M. Bishop, M.J. Bushnell and A.L. Usher, “An intelligent approach to colour recipe prediction”, JSDC, 107, pp. 235-237, 1991.

7. T. Tokunanga and Y. Honda, “CCM system utilising a neural network”, Kako Gijutsu (Dyeing \& Finishing Technology), 26 (8), pp. 553-557, 1991.

8. H.R. Kang and P.G. Anderson, "Neural network applications to the colour scanner and printer calibrations", Journal of Electronic Imaging, 1 (1), pp. 125-134, 1992.

9. S. Tominaga, “Color notation conversion by neural networks”, CRA, 18 (4), pp. 253-259, 1993.

10. H.C. Van de Hulst, "Multiple Light Scattering: Tables, Formulas, and Applications”, Academic Press (NY), 1980.

11. J.H. Nobbs, "Colour-match prediction for pigmented materials”, in Colour Physics for Industry, R McDonald (ed.), Society of Dyers and Colourists, 1997.

12. K. Funahashi, “On the approximate realization of continuous mappings by neural networks”, Neural Networks, 2, pp. 182-192, 1989. 